\title{
Trips (Thysanoptera) del racimo del banano y sus enemigos naturales en el departamento del Magdalena, Colombia
}

\section{Banana flower thrips (Thysanoptera) and their natural enemies in Magdalena, Colombia}

\author{
Mayra A. García-Sarabia', Hayder S. Mizar-Caballero², Paula A. Sepúlveda-Cano* \\ Recibido para publicación: Mayo 12 de 2015 - Aceptado para publicación: Noviembre 19 de 2015
}

\begin{abstract}
RESUMEN
El cultivo del banano es una de las actividades económicas más importantes en el departamento del Magdalena, con más de 12.000 ha cultivadas. Debido a los estándares de calidad exigidos por los mercados internacionales, es necesario reconocer y dar un manejo estricto a los problemas fitosanitarios, entre los que se registra para el departamento la presencia de trips (Thysanoptera) en los racimos. Con el fin de determinar las especies de este orden de insectos que podrían tener impacto en la producción de cultivos de banano orgánico y convencional, se realizaron muestreos de trips en los racimos de 10 lotes de cada tipo de explotación y sus enemigos naturales durante el 2011 y 2012. Todo el material se procesó y depositó en la colección de entomología del Centro de Colecciones Biológicas de la Universidad del Magdalena. Se recolectaron 2937 individuos de dos especies de trips: Frankliniella parvula y F. insularis, ambos pertenecientes a la familia Thripidae (subfamilia Thripinae). F. insularis sólo se registró en cultivos orgánicos, en donde la abundancia y diversidad de trips fue mayor, aunque sin diferencias estadísticamente significativas con respecto a los sistemas de producción convencionales. Como enemigos naturales, se encontraron especies de las familias Formicidae (Tapinoma y Camponotus), Labiidae y Vespidae (Polybia) y el hongo Metarhizium anisoplae, este último solo en cultivos orgánicos. Con el presente estudio se descarta la presencia de trips que causan el óxido o mancha roja en el departamento.
\end{abstract}

Palabras clave: Musa, plagas, depredadores, hongos entomopatógenos.

\begin{abstract}
Banana crops are one of the most important economic activities in the department of Magdalena with more than 12.000 ha of crops. Due to the quality standards required by international markets, phytosanitary problems need identification and strict management, especially thrips (Thysanoptera) problems that have been recorded in banana bunches in the department of Magdalena. In order to determine the species of this insect order that could affect banana production, sampling trips were conducted in banana bunches from 10 fields of conventional and organic crops and their natural enemies during 2011 and 2012. All the materials were processed and deposited in the Colección Entomológica del Centro de Colecciones Biológicas de la Universidad del Magdalena. 2937 specimens from two species of thrips were collected: Frankliniella parvula and $F$. insularis, both belonging to Thripidae (subfamily Thripinae) family. F. insularis was only recorded in organic fields, where the abundance and diversity of thrips was higher, although no statistically significant differences were found regarding conventional production systems. As natural enemy species, Formicidae (Tapinoma and Camponotus) Labiidae and Vespidae (Polybia) families were found, and the fungus Metarhizium anisoplae, which was found only in organic crops. The results of this study provided no evidence for the presence of thrips in the department of Magdalena that cause rust or red patch.
\end{abstract}

Key words: Musa, pest, predators, enthomopathogenic fungi.

\footnotetext{
[1] Ingeniero Agrónomo, Cra 32 \#22-08 Laboratorio de Entomología. maclau_05@hotmail.com

${ }^{[2]}$ Ingeniero Agrónomo, Cra 32 \#22-08 Laboratorio de Entomología. ing.haydermizar@hotmail.com

$[3]^{*}$ Doctora en Ciencias Agrarias, Universidad del Magdalena, Cra 32 \#22-08 Laboratorio de Entomología; correo electrónico: sepulveda_ cano@yahoo.es
} 


\section{INTRODUCCIÓN}

El cultivo de banano tiene una gran participación en la actividad económica de Colombia a través de la producción y exportación de esta fruta (Arias et al. 2004), con una contribución en las exportaciones nacionales del $35 \%$ de los productos agropecuarios sin incluir el café (AUGURA 2013). En el país las zonas productoras debanano se ubican principalmente en los departamentos del Magdalena y Guajira con 13.200 ha $y$ Antioquia con 35.100 ha (AUGURA 2013; SENA 2002). Este producto se exporta principalmente a Bélgica, Reino Unido y Estados Unidos (AUGURA 2013), mercados que se ganaron gracias a la calidad de la fruta y a que en varias plantaciones de Colombia se produce banano bajo un sistema orgánico.

La presencia de mancha roja u óxido rojo afecta el cumplimiento de las exigencias de los mercados en cuanto a la calidad del producto; en la literatura se atribuye al trips Chaetanaphothrips signipennis (Thysanoptera: Thripidae) (Haddad y Leal 1996). Según DOLE (2010) este daño es producido en la superficie de los dedos de las primeras manos del racimo e inicia en la parte basal próxima al cuello en medio de los dedos y se presenta como una escoriación de forma ovalada y variable con márgenes de color café o rojizo. Se ha sugerido la presencia de óxido rojo en las plantaciones de banano del Magdalena, sin embargo, para el país sólo se han registrado las especies de trips Frankliniella parvula, F. insularis y Actinothrips sp. (Thysanoptera: Thripidae) detectadas fundamentalmente en las primeras dos semanas de desarrollo del fruto en la zona bananera de Urabá (Antioquia), pero no relacionadas aún con daños como la mancha roja del racimo (Posada 1989; Palacio 2003 y Vergara 2007).

En este contexto, este trabajo tuvo como objetivos realizar un reconocimiento de las especies de trips asociadas al racimo de banano en el departamento del Magdalena, establecer si el tipo de explotación (orgánica y convencional) tiene efecto sobre las especies que se presentan y describir el tipo de daño que ocasionan a la fruta con el fin de verificar la presencia de óxido rojo. Adicionalmente se realizó un inventario preliminar de los enemigos naturales presentes en la zona.

\section{MATERIALES Y MÉTODOS}

Los muestreos se hicieron entre agosto de 2011 y enero de 2012 en 20 lotes comerciales, 10 correspondientes a banano tradicional y 10 a banano orgánico en los municipios de Santa Marta (corregimientos Guachaca y Buritaca) y Zona Bananera (corregimientos Río Frío, Santa Rosalía, Orihueca y Sevilla). En cada lote se seleccionaron 30 plantas al azar (separadas al menos por $10 \mathrm{~m}$ ) en estado de emergencia de la inflorescencia. En cada racimo se recolectaron durante 10 minutos los trips que se encontraran entre los dedos y las flores con un pincel delineador \#0 Corona RG595, los cuales se almacenaron en viales con alcohol al $70 \%$ y se transportaron al laboratorio de entomología de la Universidad del Magdalena. Los especímenes se aclararon en $\mathrm{KOH}$ al $10 \%$ durante 24 horas, posteriormente se lavaron con agua destilada y una gota de ácido acético, finalmente se pasaron por alcohol al 50\%, 70\% y $90 \%$ en su orden) y se montaron en placas portaobjetos con solución Hoyer. Los ejemplares se identificaron en un microscopio Leica DM750 con software Leica Application Suite ${ }^{\circledR}$ v.1.7.0; se utilizaron las claves propuestas por Mound y Kibby (1998) y Soto y Retana-Salazar (2003) y se realizó una diagnosis siguiendo la terminología propuesta por los mismos autores. Todo el material se depositó en el Centro de Colecciones Biológicas de la Universidad del Magdalena.

Para la captura de enemigos naturales, en cada muestreo se recolectaron insectos que se encontraran depredando trips, los cuales se conservaron en viales con alcohol al $70 \%$; adicionalmente los trips que se 
observaron con algún signo o síntoma de afección por patógenos (cambios en el color, movilidad y aspecto general) se almacenaron individualmente en viales secos para aislar el agente entomopatógeno asociado tras ubicar los trips en cámara húmeda.

Las variables número de trips por lote (abundancia) y número de especies de enemigos naturales se analizaron bajo un diseño completamente al azar con dos tratamientos: banano orgánico y banano convencional. Todas las comparaciones entre variables de la investigación se realizaron mediante el programa estadístico Statgraphics $\mathbb{R}_{\text {. }}$

Para descartar la presencia de patógenos en las lesiones, se tomaron los dedos afectados, se observaron al estereoscopio y se describió la lesión, y posteriormente se llevaron al laboratorio de Fitopatología de la Universidad del Magdalena en donde se realizaron cortes de tejidos afectados, se colocaron en cámara húmeda y luego se sembraron en dos medios de cultivo: Agar Nutritivo y PDA. Adicionalmente, se realizó un análisis de perfil del racimo.

\section{RESULTADOS Y DISCUSIÓN}

\section{Tisanópteros asociados al racimo de banano.}

Se recolectaron 2937 individuos de dos especies de trips: Frankliniella parvula y F. insularis, ambos pertenecientes a la familia Thripidae (subfamilia Thripinae). El género Frankliniella, se caracteriza por poseer diferentes especies de importancia económica en la agricultura de todo el mundo, como frijol ( $F$. insularis), banano y plátano ( $F$. parvula), en tomate ( $F$. schultzei) (Soto y Retana-Salazar 2003) y algodón (F. gemina) (Ortiz 1977). En nuestro país su importancia radica en que son especies polífagas y transmisoras de enfermedades virales en diferentes cultivos (Calixto 2005). El género cuenta con al menos 200 especies descritas, asociadas a flores de distintas plantas, constituyéndose de esta forma en uno de los géneros más numerosos del continente americano (Porres 2008).

Las comparaciones entre los sistemas convencional y orgánico de banano, no mostraron diferencias significativas entre la cantidad de trips en sus racimos $(P=0,0731)$, aunque se observó una tendencia a que los lotes comerciales orgánicos presentaran mayor abundancia (1733 individuos en total) y riqueza (dos especies de trips, F. parvula y F. insularis), con respecto a los lotes de explotación tradicional (abundancia 1204 especímenes y una especie, F. parvula). A pesar de estar presente en otras regiones productoras de banano en el país. F. insularis solo se registró en plantaciones orgánicas en el departamento del Magdalena, sin embargo, no hay características biológicas en la literatura que den indicios sobre esta preferencia.

Los ejemplares identificados presentaron los caracteres diagnósticos propios del género Frankliniella según Mound y Marullo (1996): cuatro pares de setas en el tórax (Figura 1A); antenas de ocho segmentos y en los segmentos III y IV se observa claramente la presencia de un sensorio bifurcado (Figura 1B); furca metaesternal sin espínula (Figura 1C).

Frankliniella parvula Hood (Thysanoptera: Thripidae).

Diagnosis. Tamaño. Longitud promedio tomada desde el ápice de la cabeza hasta el margen posterior del tubo terminal 1,37 mm (Figura 2A) Coloración. Café oscura excepto flagelómero 1, el ápice de los fémures, tibias, tarsos y alas amarillo claro y flagelómeros 2-8 café claro. Textura. Parte posterior de la cabeza con estrías transversales entre los ocelos y la cérvix; tergos del protórax, mesotórax y márgenes laterales de los segmentos abdominales suavemente reticulados; tergo metatorácico con reticulación en la mitad anterior y estrías longitudinales en la mitad posterior. Setas. Cabeza casi tan ancha como larga, cubierta con setas 

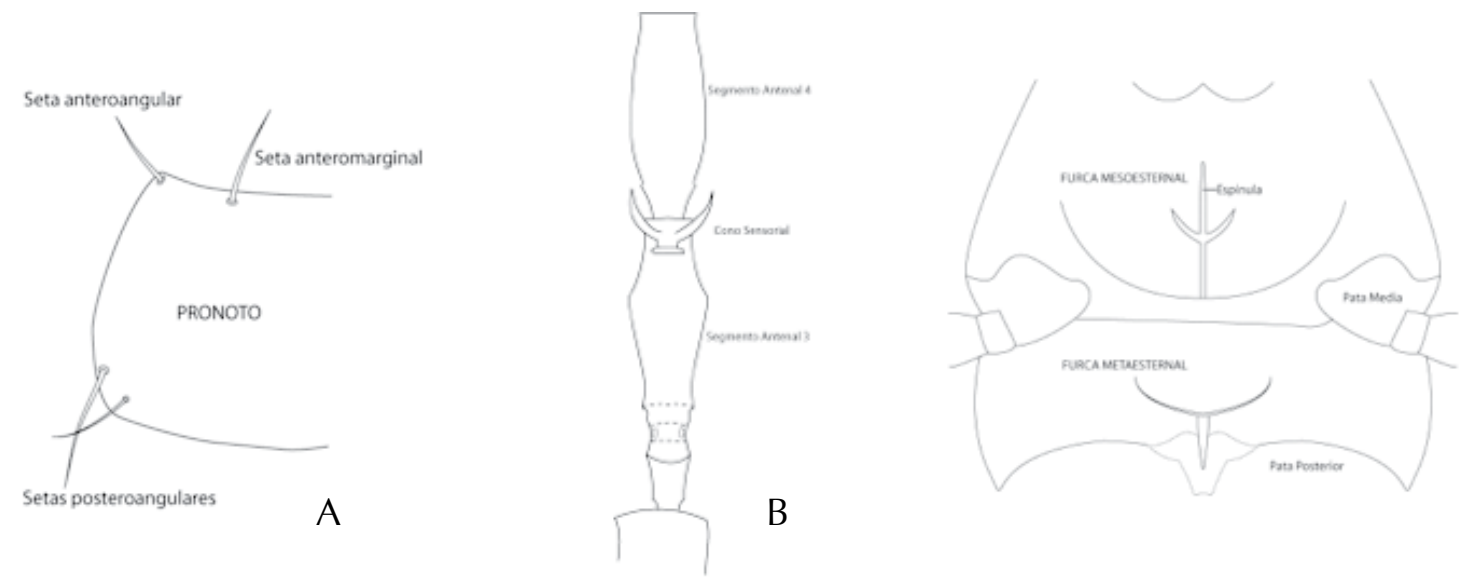

$B$

C

Fuente: Paula A. Sepúlveda-Cano

Figura 1. Caracteres diagnósticos del género Frankliniella A) Vista dorsal del pronoto B) vista dorsal de segmentos III y IV de la antena de $F$. parvula C) Vista ventral del esterno torácico señalando la posición de la furca mesoesternal y furca metaesternal de F. parvula.

dispersas incluyendo setas entre las facetas de los ojos compuestos resaltando una seta ocelar larga (entre el ocelo medio y los ocelos laterales) (Figura 2B) y dos setas postoculares. Antenómeros con setas diminutas (1/3 Diámetro Ocelar (DO)) distribuidas uniformemente y antenómeros III y IV con conos sensoriales bifurcados (Figura 1B). Tórax rectangular con setas anteroangulares (aa), anteromarginales, posteroangular epimeral y posteromarginal bien desarrolladas (Figura 1A). Alas con líneas de setas completas en cada vena (Figuras 2C).
Tenidia presentes en los terguitos $\mathrm{V}$ a VIII, en el último ubicado antes del espiráculo. Furca metaesternal presente como en la figura 1C sin espínula. Esta especie se separa fácilmente de F. insularis por tener el pedicelo del segmento antenal III muy largo, el doble del diámetro del borde sub-basal (Figura 1B).

Anotaciones biológicas: Los ejemplares de F. parvula se localizaron generalmente en la inflorescencia, y en las manos más cercanas a la bacota, ocasionando unas punturas

Fuente: Paula A. Sepúlveda-Cano
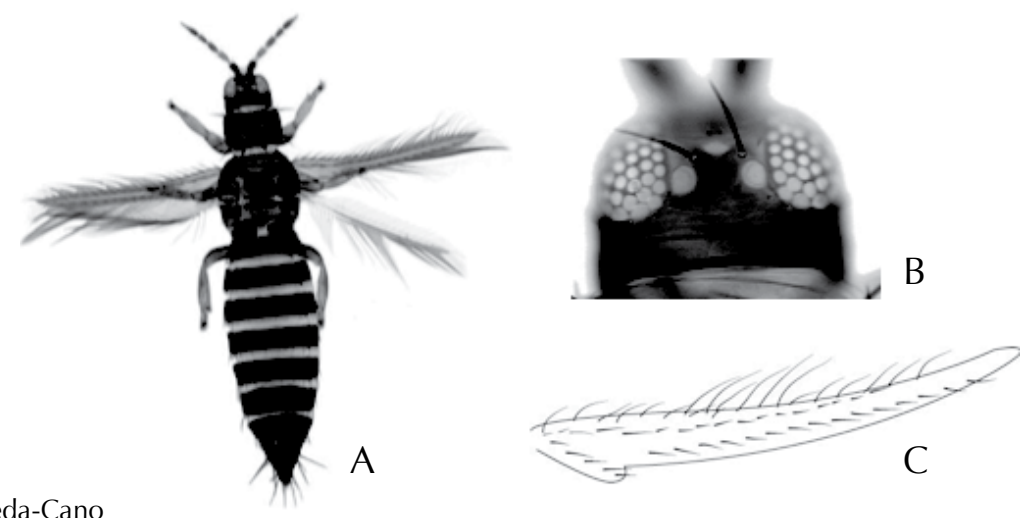

Figura 2. Características morfológicas de hembra adulta de Frankliniella parvula Hood A. Vista ventral, B. Vista ventral cabeza mostrando dos setas intraocelares, C. Ala anterior mostrando líneas de setas completas en cada vena 
inicialmente traslúcidas, que posteriormente se tornan marrón y/o rojizas en los frutos en madurez de cosecha.

\section{Frankliniella insularis Franklin (Thysanoptera: Thripidae)}

Diagnosis. Tamaño. Longitud promedio tomada desde el ápice de la cabeza hasta el margen posterior del tubo terminal 1,3 $\mathrm{mm}$ (Figura 3A). Coloración. El cuerpo y patas café oscuro, exceptuando el ápice del fémur y las tibias en las propatas y el ápice de la tibia y tarsos de las meso y metapatas, las alas y los flagelómeros III y hasta la mitad del IV - V de color más amarillo. Textura. La parte posterior de la cabeza presenta estrías transversales entre los ocelos y la cérvix; tergos del protórax, mesotórax y márgenes laterales de los segmentos abdominales suavemente reticulados, tergo metatorácico con reticulación en la mitad anterior y estrías longitudinales en la mitad posterior. Setas. Cabeza un poco más ancha que larga, con setas extendidas incluyendo setas entre las facetas de los ojos compuestos, resaltando claramente una seta larga ocelar (por fuera del ocelo medio y los ocelos laterales) (Figura 3B) y dos setas postoculares. Antenómeros con abundantes setas diminutas y entre dos y cuatros setas largas con distribución uniforme y con conos sensoriales en el segmento VI. Tórax rectangular con setas anteroangulares (aa), anteromarginales (am), posteroangular (pa), epimeral (e) y posteromarginal (pm) bien desarrolladas. Tenidia presentes en los terguitos V a VIII, y en el segmento VIII está ubicada por encima del espiráculo, peine distal en el segmento VIII interrumpido medialmente (Figura 3C).

\section{Descripción del daño}

El daño que se registró para estas dos especies en el racimo del banano correspondió a pequeñas pústulas inicialmente verde claro y luego café, ásperas al tacto, distribuidas por toda la superficie del dedo en forma aleatoria y una mayor abundancia en las últimas manos. En la evaluación de perfil de racimo, ese daño se incluye dentro de speckling de fruto causado por agentes bióticos. Las lesiones encontradas no coinciden con lo descrito para óxido rojo por Palacio (2003), lo que sugiere que las especies de trips F. parvula y F. insularis, no están relacionadas con esa lesión y que cuando las manchas de color rojizo se presentan en las plantaciones pueden ser lesiones mecánicas, efecto del roce entre frutas por causa del viento $u$ otras acciones que se consideren daño físico.
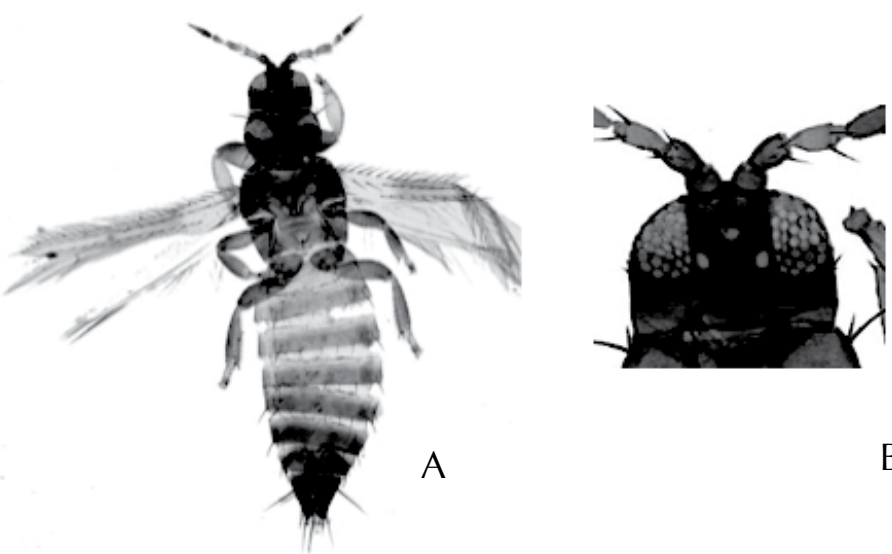

B

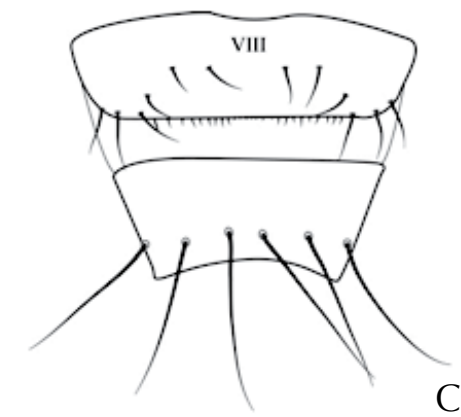

Fuente: Paula A. Sepúlveda-Cano

Figura 3. Características morfológicas de hembra adulta de Frankliniella insularis Hood A. Vista ventral, B. Vista ventral cabeza mostrando dos setas intraocelares por fuera del triángulo ocelar. C. Peine distal en el segmento abdominal VIII interrumpido medialmente. 
En el análisis de perfil de los racimos evaluados, prevalecieron otros daños no relacionados con los trips ni la mancha roja, estos daños fueron: mancha de madurez, daño corona, cuello roto, maltrato en campo, entre otros. Adicionalmente, en los análisis de perfil, las pústulas ocasionadas por trips se redujeron a medida que se desarrolló el fruto, ya que este se va engrosando y allí van generando nuevas células, lo que produce un estiramiento en la epidermis y que algunos de estos puntos se desvanezcan y los otros cicatricen tal como lo afirma Palacio (2003).

Las siembras realizadas en el laboratorio de fitopatología de la Universidad del Magdalena de cortes de tejidos en Agar Nutritivo y PDA (papa-dextrosa-agar), sólo condujeron al crecimiento de una bacteria gram positiva aun no identificada, lo que sugiere que el daño ocasionado por estos insectos puede servir de puente para que se desarrollen microorganismos en la planta como lo describe Lewis (1973).

\section{Enemigos naturales}

En cuanto a los enemigos naturales durante el trabajo se recolectaron seis especies de insectos de las familias Hymenoptera: Formicidae (Tapinoma melanocephalum., Pheidole sp. y Camponotus sp.), Dermaptera: Labiidae (género sin determinar) e Hymenoptera: Vespidae (Polybia sp. y Polistes erythrocephalus), y una especie de hongo Sordariomycete de la familia Clavicipitaceae (Metarhizium anisopliae). Varios trabajos han documentado los hábitos de los enemigos naturales que se encontraron en estos muestreos, los cuales en su totalidad han sido previamente registrados como depredadores y patógenos de otras especies de insectos, y que algunos podrían tener potencial en programas de manejo integrado de trips en cultivos de banano (Ej. King y Saunders 1984; CORPOICA 1999; ICA 2012; Lemos et al. 2003; Nogueira y Gordon 2004; Pérez et al. 2004, Cañellas et al. 2005; Fernández y Sharkey 2006; Abramson et al. 2007; Orduño
2009; Pirk et al. 2009; Soler 2004 y Vélez et al. 2006).

En general, las hormigas se encontraron con mayor frecuencia que otros biocontroladores. Algunos autores han registrado que varias especies de hormigas tienen hábitos depredadores y pueden alimentarse de insectos que afectan la calidad comercial de productos agrícolas (Gallego y Armbrecht 2005; Mera et al. 2010). Sin embargo, se requieren evaluaciones sobre la relación entre las especies de hormigas y trips presentes en el racimo del banano, así como la interacción con otros elementos del agroecosistema bananero, con el ánimo de determinar la distribución de nidos en campo, sus preferencias y de diseñar estrategias para su conservación y manejo. En cuanto a M. anisopliae, es tal vez uno de los agentes de control con mayor potencial para las plantaciones de banano del departamento del Magdalena, dado que se ha registrado con éxito en otras especies de trips (Ekesi et al. 1998; Maniania et al. 2003; Ansaria et al. 2007) y se multiplica con relativa facilidad, incluso los mismos agricultores pueden hacerlo en sus fincas.

Durante las evaluaciones fue evidente la mayor abundancia de enemigos naturales en fincas orgánicas (Tabla 1) y que algunas especies fueron exclusivas para estos ecosistemas, aunque no se encontraron diferencias estadísticamente significativas $(P=0,07931)$. Existen investigaciones que sugieren que la mayor abundancia de enemigos naturales en los cultivos orgánicos se debe principalmente a que la diversidad vegetal (en especial la presencia de arvenses) juega un papel importante $\mathrm{e}$ influye de manera destacada en la abundancia, diversidad y dinámica de los insectos benéficos (Blanco y Leyva 2007).

De acuerdo con los resultados de este trabajo se recomienda a todas las empresas productoras de banano e investigadores desarrollar estudios 
Tabla 1. Especies de enemigos naturales de trips registradas en 10 lotes comerciales de banano tipo convencional y 10 lotes comerciales tipo orgánico en seis corregimientos del departamento del Magdalena.

\begin{tabular}{lcc}
\hline \multicolumn{1}{c}{ Especie } & $\begin{array}{c}\text { Lotes en donde se presentó } \\
\text { el enemigo natural } \mathbf{\%}\end{array}$ & Tipo de explotación \\
\hline Tapinoma melanocephalum & 20 & Orgánico y Convencional \\
Camponotus sp. & 15 & Orgánico \\
Pheidole sp. & 5 & Orgánico \\
Polistes erythrocephalus & 60 & Orgánico y Convencional \\
Polybia sp & 5 & Orgánico y Convencional \\
Labiidae & 5 & Orgánico \\
Metarhizium anisioplae & 40 & Orgánico \\
\hline
\end{tabular}

complementarios en otras fincas productoras de banano del departamento del Magdalena para determinar la presencia de otras especies de Tisanópteros y para hacer seguimiento a las prácticas agronómicas y factores ambientales que permitan determinar $y / 0$ esclarecer el origen al daño conocido como óxido rojo y si este tiene alguna relación con estos insectos.

\section{CONCLUSIONES}

Las lesiones que los agricultores reconocieron como óxido rojo para la época de las evaluaciones, podrían no ser originadas por $C$. signipennis, ya que la especie no se recolectó en ninguna de los lotes evaluados que presentaron los síntomas.

Existe una diversidad de especies de enemigos naturales que deberían ser protegidas en las plantaciones por ser depredadores y/o patógenos de Frankliniella insularis y F. párvula.

\section{Agradecimientos}

Los autores desean agradecer a la Universidad del Magdalena por su apoyo logístico, así como a todos los productores de banano del departamento del Magdalena que permitieron realizar los muestreos en campo. Igualmente agradecer al fitopatólogo Alberto Páez Redondo por su asesoría en el reconocimiento de microorganismos. El último autor agradece al profesor Rodrigo Vergara, por sus aportes al inicio de este trabajo.

\section{BIBLIOGRAFÍA}

Abramson, C., Wanderley, P., Mina, A. y Wanderley, M. 2007. Capacity of earwig Marava arachidis (Yersin) to access fennel plants Foeniculum vulgare Mill in laboratory and field. Ciencia Rural 37:1524-1528.

Ansaria, M., Shaha, F., Whittakerb, F., Prasadc, M. y Butta, T. 2007. Control of western flower thrips (Frankliniella occidentalis) pupae with Metarhizium anisopliae in peat and peat alternative growing media. Biological control 40(3):293-297.

Arias, P., Dankers, C., Liu, P. y Pilkauskas, P. 2004. La economía mundial del banano 1985-2002. Organización de las naciones unidas para la agricultura y la alimentación-FAO, Roma, 96p.

AUGURA-Asociación de Bananeros de Colombia. 2013. Coyuntura bananera 
Colombiana 2012. Ed. Comunicaciones AUGURA, 32p.

Blanco, Y. y Leyva, A. 2007. Las arvenses en el agroecosistema y sus beneficios agroecológicos como hospederas de enemigos naturales. Cultivos Tropicales 28(2):21-28.

Calixto, C. 2005. Trips del suborden Terebrantia (Insecta: Thysanoptera) en la Sabana de Bogotá. Revista Colombiana de Entomología 31(2):207-213.

Cañellas, N., Piñol, J. y Espadaler, X. 2005. Las tijeretas (Dermaptera, Forficulidae) en el control del pulgón en cítricos. Boletín Sanidad Vegetal y Plagas 31:161-169.

CORPORACIÓN COLOMBIANA DE INVESTIGACIÓN AGROPECUARIA (CORPOICA).1999. UsodeTrichogramma sp. y Polistes erythrocephalus en el manejo biológico de plagas lepidópteras. Programa Nacional de transferencia de tecnología Agropecuaria, Palmira-Valle (Colombia), 6p.

DOLE. 2010. Manual de calidad defectos y tolerancias. DOLE, Bogotá, 26p.

Ekesi, S., Maniania, N., Ampong-Nyarko, K y Onu, I. 1998. Potential of the entomopathogenic fungus, Metarhizium anisopliae (Metsch.) Sorokin for control of the legume flower thrips, Megalurothrips sjostedti (Trybom) on cowpea in Kenya. Crop protection 17(8):661-668.

Fernández, F. y Sharkey, M. 2006. Introducción a los Hymenoptera de la Región Neotropical. Sociedad Colombiana de Entomología, Bogotá D. C.894p.

Gallego, M. y Ambretcht, I. 2005. Depredación por hormigas sobre la broca del café Hypothenemus hampei (Curculionidae:
Scolytinae) en cafetales cultivados bajo dos niveles de sombra en Colombia. Manejo Integrado de Plagas y Agroecología 76: 32-40.

Haddad, O. y Leal, F. 1996. Situación actual y perspectivas de la producción de cambur de exportación y otras Musáceas en el estado Aragua. Papeles de Fundacite Aragua, 41p.

ICA, 2012. Manejo fitosanitario del cultivo del algodón (Gossypium hirsutum) - Medidas para la temporada invernal. Instituto Colombiano Agropecuario, 43p.

King, A. y Saunders, J. 1984. Las plagas invertebradas de cultivos anuales alimenticios en America Central. Londresoverseas devel: ADM, 182p.

Lemos, W., Ramalho, F. y Zanuncio, J. 2003. Age-Dependent Fecundity and LifeFertility Tables for Euborellia Annulipes (Lucas) (Dermaptera: Anisolabididae) A Cotton Boll Weevil Predator in Laboratory Studies with An Artificial Diet. Environmental Entomology 32(3):592601.

Lewis, T. 1973. Thrips, their biology, ecology and economic important. Academic press of London and New York, 348p.

Maniania, N., Sithanantham, S., Ekesi, S., Ampong-Nyarko, K., Baumgärtner, J., Löhr, B. y Matoka, C. 2003. A field trial of the entomogenous fungus Metarhizium anisopliae for control of onion thrips, Thrips tabaci. Crop Protection 22(3): 553559.

Mera, Y., Gallego, M. y Armbrecht, I. 2010. Interacciones entre hormigas e insectos en follaje de cafetales de sol y sombra, Cauca-Colombia. Revista Colombiana de Entomología 36 (1): 116-126. 
Mound, L. y Marullo, R. 1996. The thrips of Central and South America: An introduction (Insecta: Thysanoptera). Memoirs on Entomology International, $487 p$.

Mound L. y Kibby, G. 1998. Thysanoptera an Identification Guide. Second Edition, CAB International, 67p.

Nogueira, M. y Gordon, H. 2004. Predaceous Ant Fauna in New Sugarcane Fields in the State of São Paulo, Brazil. Brazilian Archives of Biology and Technology 47(5): 805-811.

Orduño, N. 2009. Virulencia de Beauveria bassiana y Metarhizium anisopliae sobre el picudo del nopal Metamasius spinolae. México, 2009. 68p. tesis de maestría, Institución de enseñanzas e investigación en ciencias agrícolas.

Ortiz, M. 1977. El género Frankliniella Karny (Thysanoptera: Thripidae) en el Perú. Revista Peruana de Entomología 20(1): 49-62

Palacio, M. 2003. Contribuciones al conocimiento de los orígenes y causas de la mancha roja del banano en la zona de Urabá. Tesis de Ingeniería Agronómica, Universidad Nacional de Colombia Sede Medellín, Medellín.

Pérez, O., González, R., Bisset, J., Navarro, A., Hernández, N. y Martínez, A. 2004. Efectos de Tapinoma melanocephalum (Hymenoptera: Formicidae) sobre huevos de Aedes aegypti (Diptera: Culicidae). Revista Cubana de Medicina Tropical 56(3): 167-171.

Pirk, G., Di Pasquo, F. y López, J. 2009. Diet of two sympatric Pheidole spp. ants in the central Monte desert: implications for seed-granivore interactions. Insectes
Sociaux 56:277-283.

Porres, V. 2008. Inventario de especies de trips (Insecta: Thysanoptera) del género Frankliniella asociadas a los cultivos de las regiones centro y occidente de Guatemala y su distribución geográfica. Tesis Ingeniero Agrónomo, Universidad Del Valle De Guatemala, Guatemala.

Posada, O. 1989. Lista de insectos dañinos y otras plagas en Colombia. Bogotá: Instituto Colombiano Agropecuario, $662 \mathrm{p}$.

SENA- Servicio nacional de aprendizaje. 2002. Caracterización del subsector bananero en Colombia, 136p.

Soler, J. 2004. Invasiones de hormigas. Animalia XVII(167): 64-66.

Soto, G. y Retana-Salazar, A. 2003. Clave ilustrada para los géneros de Thysanoptera y especies de Frankliniella presentes en cuatro zonas hortícolas en Alajuela, Costa Rica. Agronomía Costarricense 27:55- 68 .

Vélez, M., Bustillo, A. y Posada, J. 2006. Depredación de Hypothenemus hampei por hormigas durante el secado solar del café. Revista Cenicafé (Colombia). 56(3):198-207.

Vergara, R. 2007. Manejo integrado de insectos asociados al racimo del banano. En: Memorias XXXIV Congreso Sociedad Colombiana de Entomología. Cartagena de Indias, Colombia. p68-86. 